\title{
Modular bioinformatics analysis demonstrates that a Toll-like receptor signaling pathway is involved in the regulation of macrophage polarization
}

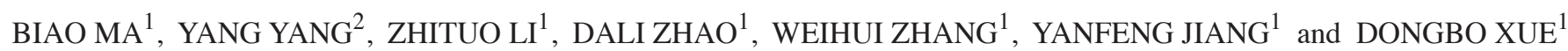 \\ Departments of ${ }^{1}$ General Surgery, and ${ }^{2}$ Obstetrics and Gynecology, \\ The First Affiliated Hospital of Harbin Medical University, Harbin, Heilongjiang 150001, P.R. China
}

Received January 16, 2018; Accepted August 24, 2018

DOI: $10.3892 / \mathrm{mmr} .2018 .9486$

\begin{abstract}
In recent years, an increasing number of studies on the roles of macrophages in tumors, immune responses and metabolism have been published, in which macrophage polarization has been an extensively discussed topic. In the present study, differentially expressed genes in various types of macrophages were analyzed using the Gene Expression Omnibus database. Cluster analysis of differentially expressed genes was conducted, and a protein-protein interaction (PPI) network was constructed. Finally, modular analysis and functional enrichment analysis revealed that a Toll-like receptor (TLR) signaling pathway is involved in the regulation of macrophage polarization. Furthermore, the high-degree proteins in the PPI network that are involved in the molecular regulation of macrophage polarization are closely associated with proteins of the TLR signaling pathway. These results suggested that the TLR signaling pathways may be a principal direction of future research on the regulation of macrophage polarization.
\end{abstract}

\section{Introduction}

Macrophages are the primary effector cells of the immune system and are involved in inflammatory and anti-infective responses (1). They additionally serve an important role in tissue homeostasis, promote the growth of cells in tissues and are involved in the repair of tissue damage (2). Macrophages undergo different forms of polarization under the action of different inducers and are polarized to M1 or M2

Correspondence to: Dr Yanfeng Jiang or Dr Dongbo Xue, Department of General Surgery, The First Affiliated Hospital of Harbin Medical University, 23 Youzheng Street, Harbin, Heilongjiang 150001, P.R. China

E-mail: 115838744@qq.com

E-mail: xue9971@sina.com

Key words: Toll-like receptor signaling pathway, modular bioinformatics analysis, macrophage polarization macrophages (3). M1 macrophages are additionally known as classical pathway-activated macrophages. They are primarily induced by bacterial products and $\mathrm{T}$ helper (Th)1-type cytokines, including interferon (IFN) $-\gamma$. Their characteristic effects include eliminating intracellular microorganisms and producing large quantities of pro-inflammatory mediators (4). M2 macrophages are additionally termed alternative pathway-activated macrophages (5). The primary inducers of M2 macrophages are Th2-type cytokines [interleukin (IL)-4, IL-13 and IL-10], glucocorticoids and immunoglobulin complexes and Toll-like receptor (TLR) ligands (6). The principal effect of M2 macrophages is the suppression of inflammatory responses (7). Macrophage polarization commonly occurs during the pathogenesis and progression of inflammatory diseases, including cancer, obesity and cardiovascular diseases, and has a guiding significance for the prognosis of specific tumors (8-11).

In the present study, a protein-protein interaction (PPI) network was constructed based on differentially expressed genes in macrophages of different polarization types from the Gene Expression Omnibus (GEO) database. The aim of the present study was to identify important signaling pathways and gene groups that are involved in the regulation of macrophage polarization through modular analysis and functional enrichment analysis, which may provide specific novel insight for the treatment of human immune-associated diseases.

\section{Materials and methods}

Gene expression profiles of human M1 and M2 macrophages. Gene expression profiles of human M1 and M2 macrophages were downloaded from the GEO database [https://www. ncbi.nlm.nih.gov/geo/; accession nos. GSE18686 (12) and GSE35449 (13)]. Expression profile data of GSE18686 and GSE35449 were obtained from M1 and M2 macrophages cultured with inducer or without inducer, respectively $(12,13)$. The GSE18686 and GSE35449 data sets were tested using the Illumina HumanHT-12 v3.0 Gene Expression BeadChip platform (Illumina, Inc., San Diego, CA, USA).

The data of M1 macrophages treated with lipopolysaccharide (LPS) and IFN- $\gamma$, M2 macrophages cultured with IL-4 and the corresponding control group (M0 macrophages) 
were selected from GSE18686, with six biological replicates included in each group. The data of the M1 and M2 macrophage groups in addition to the control group were selected from GSE35449, and seven biological replicates were included in each group.

Pretreatment of expression profile data. GSE18686 and GSE35449 expression matrix data sets were downloaded and pretreated using the quantile method in the lumi software package v2.32.0 (14,15). The mean value of the different probes that mapped to the same gene (GeneSymbol) was calculated. The GSE18686 data set consists of 48,802 probes, and a final total of 19,489 genes remained following the treatment. The GSE35449 data set consists of 48,797 probes, and 19,487 genes remained following the treatment. The gene expression data sets were extracted for M1 and M2 macrophages, according to the aforementioned methods.

Detection of differentially expressed genes. The limma package in Bioconductor (v3.36.2) (16) was employed to analyze genes that were differentially expressed between M1 and M0 or between M2 and M0 in GSE18686 and GSE35449. This method is comprised of the following steps: i) Constructing a design matrix for the preprocessed data; ii) estimating the number of folds in differential gene expression using a linear model; iii) followed by using a Bayesian approach for smoothing the standard deviation (17); and iv) finally utilizing different parameters for output of the differentially expressed genes. The $\log 2$ of fold change $(\log 2 \mathrm{FC})$ and P-values were used as parameters for the selection of differentially expressed genes; $\mathrm{P}<0.05$ was considered to indicate a statistically significant difference, determined using a Student's t-test, and an absolute value of $\log 2 \mathrm{FC} \geq 1$ (the differential expression coefficient was 2). Subsequently, the differentially expressed genes in the intersection of GSE18686 and GSE35449 were selected.

Cluster analysis of differentially expressed genes in intersection. The gplots software package (v2.17.0) (18) was employed to calculate and construct thermal graphs from the cluster analysis of the differentially expressed genes in M1 (Fig. 1A and B) and M2 (Fig. 1C and D) in the intersection of GSE18686 and GSE35449. The expression values of these differentially expressed genes were analyzed for hierarchical clustering.

Modular analysis of differentially expressed genes in the intersection. To further analyze the differentially expressed genes at the molecular level, Search Tool for the Retrieval of Interacting Genes/Proteins v9.1 (https://string-db.org/) was used to obtain information regarding PPI pairs based on the differentially expressed genes in the GSE18686 and GSE35449 intersection and Cytoscape (v3.4.0) (19) to construct PPI networks. To obtain functional modules in the PPI networks, communities in the PPI networks were extracted using CFinder (v2.0.6) (20).

Functional enrichment analysis of each community. The candidate genes of each community were submitted to the Database for Annotation, Visualization and Integrated Discovery database (http://david.abcc.ncifcrf.gov/), and the complete genome of Homo sapiens was used for assessment and comparison as background genes. The 'Functional Annotation Tool' was used to obtain the Kyoto Encyclopedia of Genes and Genomes (KEGG; https://www.genome.jp/kegg/) pathway enrichment analysis results (the P-value cutoff was $0.05)$.

\section{Results}

Identification of differentially expressed genes in gene expression profiles. A total of 338 genes that were differentially expressed between the M1 test group and the M0 control group were obtained from GSE18686 when $\mathrm{P}<0.05$ and the absolute $\log 2 \mathrm{FC}$ value was $\geq 0.58$; whereas, 636 genes that were differentially expressed between the M1 test group and the M0 control group were obtained from GSE35449 (Table I). A total of 151 differentially expressed genes were obtained from the intersection of differentially expressed genes from GSE18686 and GSE35449 between the M1 test group and the M0 control group (data not shown). Within the same threshold range as above, 273 genes that were differentially expressed between the M2 test group and the M0 control group were obtained in GSE18686; whereas, 1,171 genes that were differentially expressed between the M2 test group and the M0 control group were identified in GSE35449 (Table I). A total of 144 differentially expressed genes were obtained from the intersection of differentially expressed genes from GSE18686 and GSE35449 between the M2 test group and the M0 control group.

Cluster analysis of differentially expressed genes in intersection. The thermal graphs from the cluster analysis of the 151 differentially expressed genes between the M1 and M0 groups in the intersection of GSE18686 and GSE35449 are demonstrated in Fig. 1A and B. The majority of the differentially expressed genes in the intersection were upregulated in the M1 group when compared with their expression levels in the M0 samples. The thermal graphs from the cluster analysis of the 144 differentially expressed genes between the M2 and M0 groups in the intersection of GSE18686 and GSE35449 are demonstrated in Fig. 1C and D. The majority of the differentially expressed genes in the intersection were upregulated in the M2 group when compared with their expression levels in the M0 samples.

Construction of PPI networks based on differentially expressed intersection genes. The PPI network based on the intersection of the differentially expressed genes between the M1 and M0 groups was constructed. This network consists of 94 protein nodes and 523 PPIs. As demonstrated in the network diagram, the proteins encoded by these intersecting differentially expressed genes exhibit a complex association. In this PPI network, the 10 highest degree proteins were signal transducer and activator of transcription $(\mathrm{STAT}) 1$ (degree $=41)$, guanylate-binding protein $(\mathrm{GBP}) 1$ (degree=35), GBP5 (degree=35), C-X-C motif chemokine $10($ CXCL10; degree $=35)$, interferon-induced protein with tetratricopeptide $($ IFIT) 2 (degree $=32)$, interferon regulator factor $($ IRF)7 (degree=32), IFIT3 (degree=32), ubiquitin-like protein ISG15 (ISG15; degree=31), IRF1 (degree=31) and interferon-induced helicase $\mathrm{C}$ domain-containing protein 1 
A
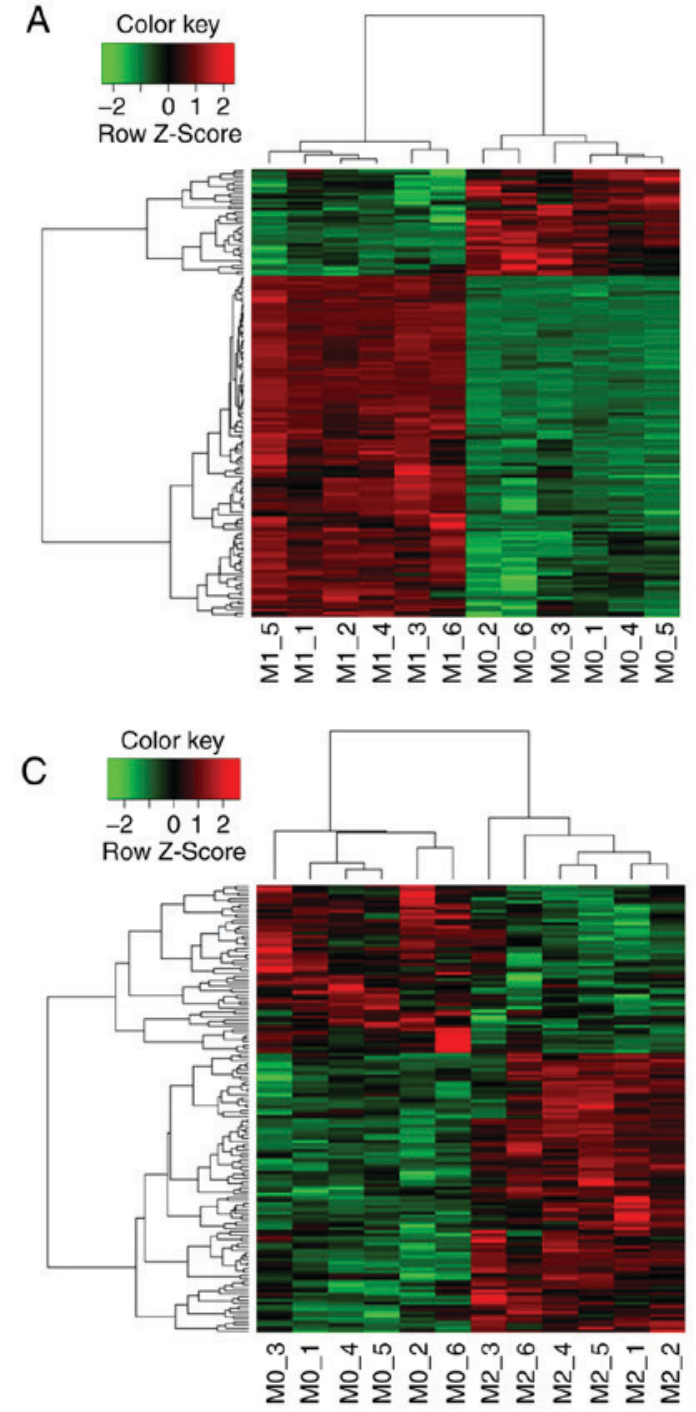

B
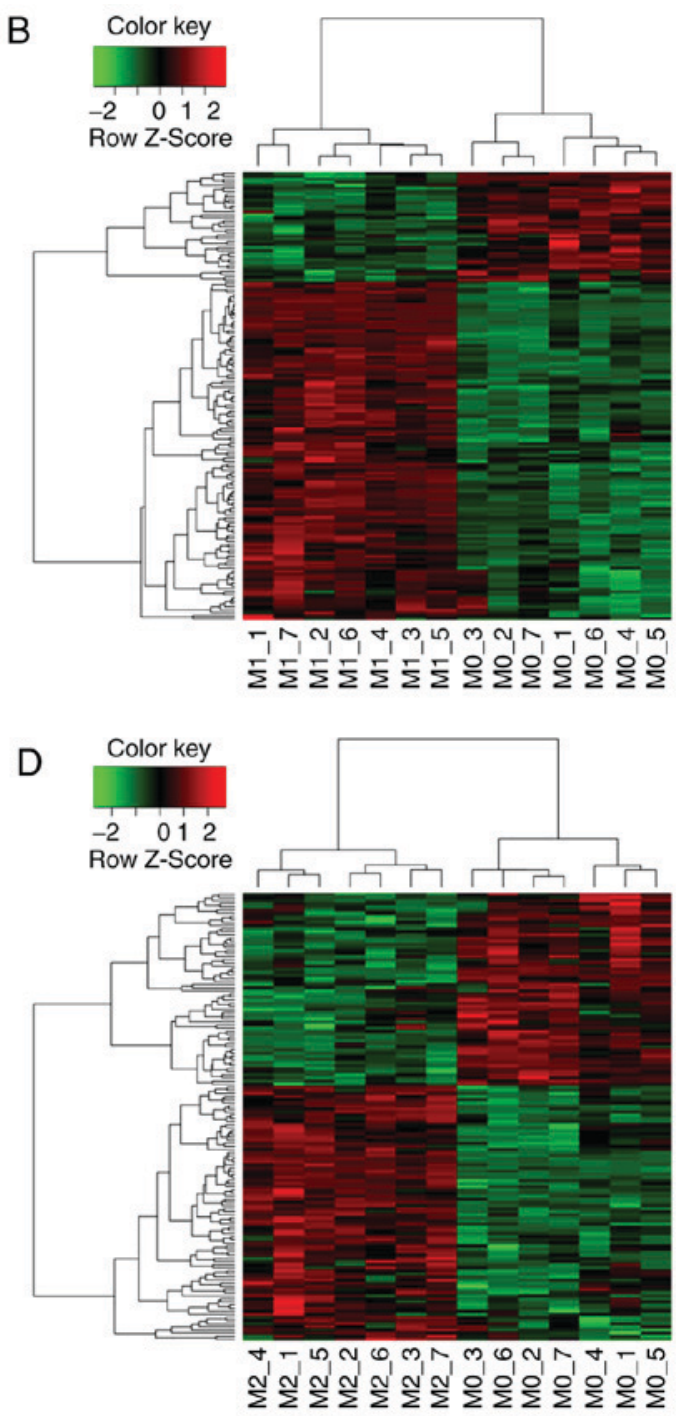

Figure 1. Cluster analysis of intersecting differentially expressed genes. (A) Thermal graph from cluster analysis of the 151 intersecting differentially expressed genes of GSE18686 in the M1 vs. M0 group. (B) Thermal graph from cluster analysis of the 151 intersecting differentially expressed genes of GSE35449 in the M1 vs. M0 group. (C) Thermal graph from cluster analysis of the 144 intersecting differentially expressed genes of GSE18686 in the M2 vs. M0 group. (D) Thermal graph from cluster analysis of the 144 intersecting differentially expressed genes of GSE35449 in the M2 vs. M0 group. Red indicates upregulated gene expression and green indicates downregulated gene expression. Each column in the thermal graph represents a sample and each row represents a gene.

(IFIH1; degree=2; Fig. 2). These 10 proteins are significant nodes in the network. A previous study demonstrated that IFN signaling activates the IRF/STAT signaling pathways through STAT1, leading to the transformation of macrophages to the M1 type (21).

The PPI network based on the intersection of the differentially expressed genes between the M2 and M0 groups is demonstrated in Fig. 3. This network consists of 56 protein nodes and 72 PPIs. The 10 highest degree proteins in this PPI network were monocyte differentiation antigen CD14 (CD14; degree $=8$ ), arachidonate 5-lipoxygenase-activating protein (ALOX5AP; degree=8), myeloid differentiation primary response gene 88 (MYD88; degree=7), cyclin-dependent kinase inhibitor 1A (CDKN1A; degree=6), protein S100-A9 (S100A9; degree=32), cytochrome b-245 heavy chain (CYBB; degree=6), cyclin-A2 (CCNA2; degree=5), protein S100-A8 (S100A8; degree=5), thymidine kinase, cytosolic (TK1; degree $=5$ ) and TLR7 (degree $=5$ ).
Modular analysis and KEGG functional analysis of each module. In the M1 vs. M0 group, communities in the PPI network were identified using CFinder software. When $\mathrm{K}=5$, three communities were identified, and there were 432,15 and 14 pairs of interacting genes in the first, second and third communities, respectively.

In the M2 vs. M0 group, communities in the PPI network were identified using CFinder software. When $\mathrm{K}=3$, five communities were identified, and there were $23,3,5,3$ and 12 pairs of interacting genes in the first, second, third, fourth and fifth communities, respectively (data not shown).

The results from KEGG functional analysis of each module are demonstrated in Fig. 4. Enrichment was obtained in community 1 of the M1 group and in communities 1,3 and 5 of the M2 group. Community 1 of the M1 group and communities 1 and 3 of the M2 group demonstrated functions primarily enriched in the 'toll-like receptor signaling pathway'. Detailed information regarding gene-interaction pairs and the degree of gene nodes in these three communities is presented in Fig. 5. 
Table I. Differentially expressed genes between M1 experimental group and M0 control group.

\section{A, GSE18686}

\begin{tabular}{lccr}
\hline Contrast group & $\begin{array}{c}\text { Number of } \\
\text { differentially expressed genes }\end{array}$ & $\begin{array}{c}\text { Number of } \\
\text { upregulated genes }\end{array}$ & $\begin{array}{c}\text { Number of } \\
\text { downregulated genes }\end{array}$ \\
\hline M1 vs. M0 & 338 & 249 & 89 \\
M2 vs. M0 & 273 & 181 & 92 \\
\hline
\end{tabular}

\section{B, GSE35449}

\begin{tabular}{lccc}
\hline Contrast group & $\begin{array}{c}\text { Number of } \\
\text { differentially expressed genes }\end{array}$ & $\begin{array}{c}\text { Number of } \\
\text { upregulated genes }\end{array}$ & $\begin{array}{c}\text { Number of } \\
\text { downregulated genes }\end{array}$ \\
\hline M1 vs. M0 & 636 & 298 & 338 \\
M2 vs. M0 & 1,171 & 399 & 772 \\
\hline
\end{tabular}

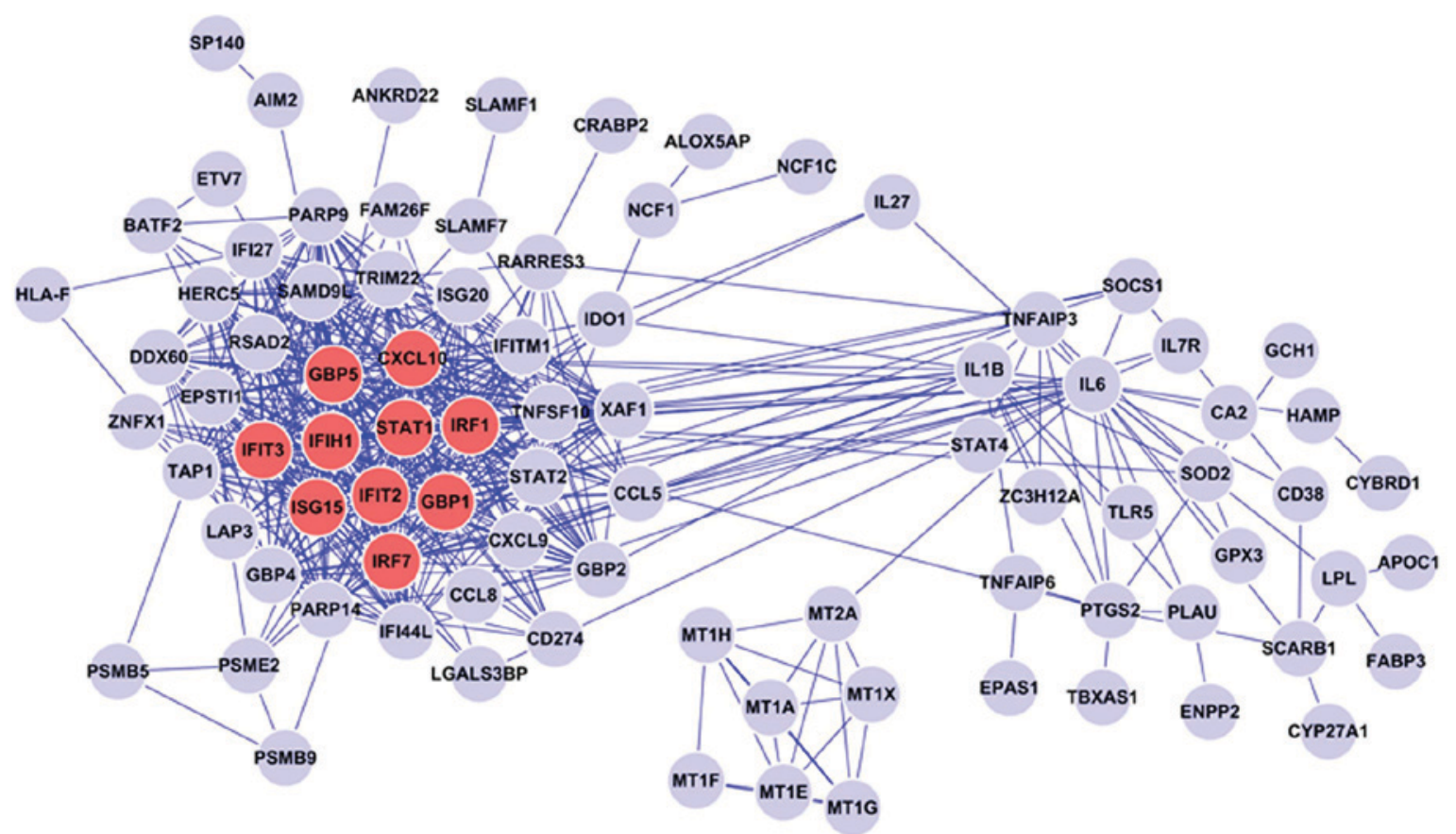

Figure 2. PPI network constructed based on the genes with differential expression between the M2 and M0 groups in the intersection of GSE18686 and GSE35449. The network is undirected, where a circular node represents a protein and a gray line represents an interaction between nodes. Red nodes indicate proteins with the highest degree in the PPI network. PPI, protein-protein interaction.

\section{Discussion}

Macrophages are multifunctional cells that perform different functions depending on the type and state of the tissues where they reside. Dysregulation of macrophage functions may lead to numerous diseases, including infectious diseases and immune disorders. In addition, macrophages serve a role in the destruction of endocrine pancreatic-cells in the autoimmune response of type 1 diabetes (21), metabolic diseases (22) and malignancies (23). The transition between macrophage polarization types serves a significant and pivotal role in the progression of these diseases (24). Therefore, the identification of the molecules and molecular groups associated with the dynamic processes of macrophages is crucial for the elucidation of the molecular basis of disease progression and the design of novel macrophage-based therapeutic strategies.

The concept of macrophage polarization types has been described as a dynamic, stepwise and continuous process of alteration from M1 to M2 (25-27). Macrophage polarization is classified into M1 (classical) and M2 (alternative) types, which is currently an intense area of research in the study of macrophage function $(3,28)$. The present study employed gene expression profiling data of M1 and M2 macrophages in the GEO database to evaluate protein-protein interrelationships and construct PPI networks through a more efficient method based on protein semantic similarity (29). However, 


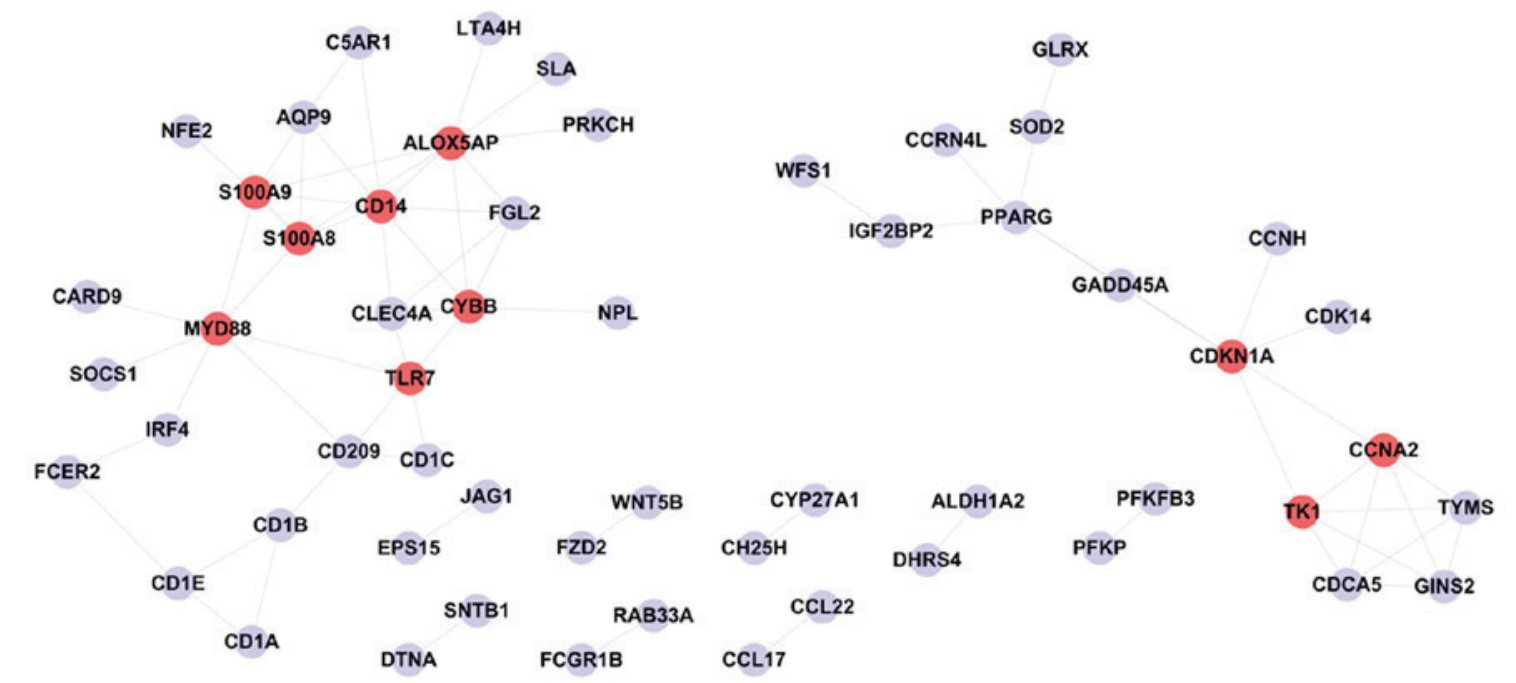

Figure 3. The PPI network based on the interaction of the differentially expressed genes between the M2 and M0 groups. This network consists of 56 protein nodes and 72 PPIs. The 10 highest degree proteins in this PPI network are presented as red nodes, where a circular node represents a protein and a gray line represents an interaction between nodes. PPI, protein-protein interaction.

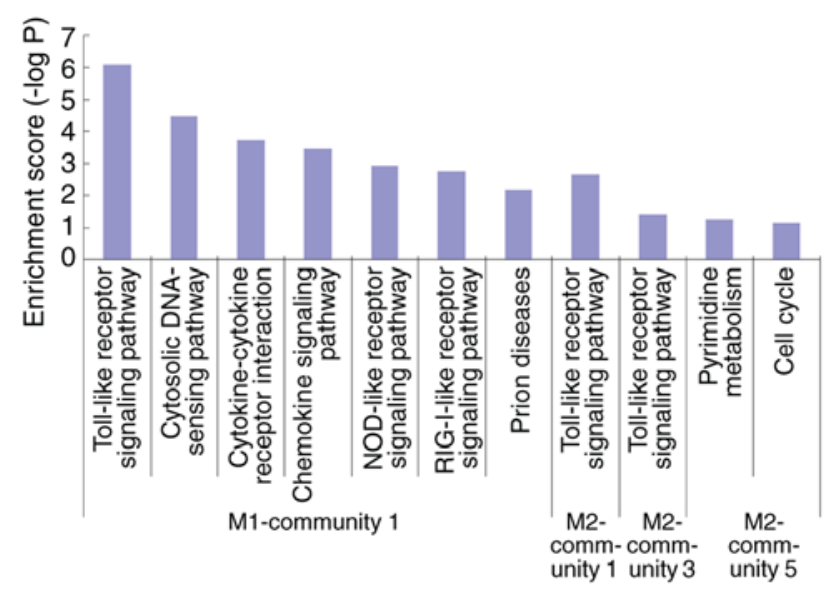

Figure 4. Kyoto Encyclopedia of Genes and Genomes enrichment analysis of the three communities of the M1 group and the five communities of the M2 group. The functions of community 1 of the M1 group and communities 1 and 3 of the M2 group were primarily enriched in Toll-like receptor signaling pathway proteins. NOD, nucleotide oligomerization domain.

it is critical to identify functional modules in the PPI network for analysis with the aim of identifying cell functions (30). The subsequent modular and KEGG enrichment analyses of each module demonstrated that the majority of the M1 and M2 modules were primarily involved in the TLR signaling pathway, suggesting that this pathway is involved in the regulation of macrophage M1 and M2 polarization.

Macrophages are the sensing cells of the immune system and are crucial mediators of the inflammatory response. TLRs, which serve an important role in the body's defense against specific pathogenic microorganisms, are the best-characterized inducers of acute inflammation (31). The initial characterization of enhancers involved in LPS-inducible gene expression in macrophages is based on the ability of stimulus-activated translational factors, including NF- $\mathrm{B}$ and IRFs (32). The TLR/NF- $\mathrm{BB}$ and Janus kinase/STAT signaling pathways (33) are involved in the regulation of macrophage polarization.
In the majority of cases, the TLR/NF- $\kappa \mathrm{B}$ signaling pathway promotes M1-type macrophage polarization when external microorganisms invade. However, this signaling pathway may additionally select the type of macrophage polarization based on the subunit composition of $\mathrm{NF}-\kappa \mathrm{B}$. When $\mathrm{NF}-\kappa \mathrm{B}$ is activated in the form of p65/p50, macrophages increase pro-inflammatory cytokine production (34), and M1 macrophages are formed. When $\mathrm{NF}-\kappa \mathrm{B}$ is activated in the form of p50/p50, M2 macrophages are formed, which occurs in tumor-associated and LPS-tolerant macrophages (35). In addition, TLR serves an important role in the regulation of post-transcriptional polarization of macrophages.

In the present study, the macrophage polarization regulatory gene profile was efficiently extracted from the accessible online database and a PPI network map of M1 and M2 macrophages, based on genes demonstrating significantly upregulated expression identified by cluster analysis of differentially expressed genes was constructed. The network map demonstrated that the first 10 high-degree proteins in the PPI network of the M1-type polarized macrophages were STAT1, GBP1, GBP5, CXCL10, IFIT2, IRF7, IFIT3, ISG15, IRF1 and IFIH1. A PPI network map of M2-type polarized macrophages was constructed using the same method, and the first 10 high-degree proteins were CD14, ALOX5AP, MYD88, CDKN1A, S100A9, CYBB, CCNA2, S100A8, TK1 and TLR7. The majority of the high-degree proteins in the PPI network that are involved in the molecular regulation of macrophage polarization are closely associated with proteins involved in the TLR signaling pathway. It was identified that the TLR7 signaling pathway serves an important role in the regulation of macrophage polarization. These results are consistent with a previous study, which demonstrated that the TLR family regulates $\mathrm{M} 1$ and M2 differentiation, in addition to demonstrating the potential importance of TLR7 in virus-induced M2 polarization (36). Furthermore, other high-degree proteins identified during comparison not only help suggest the modulating role of the TLR pathway during macrophage polarization; however, may additionally function individually 
A

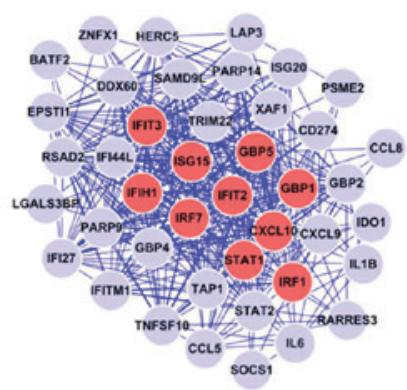

B

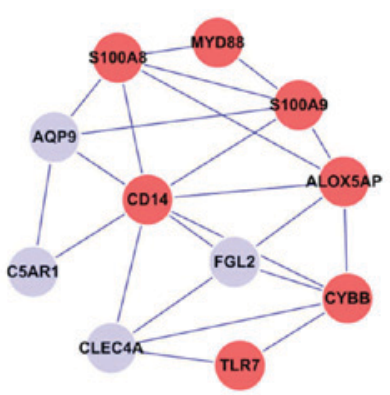

C

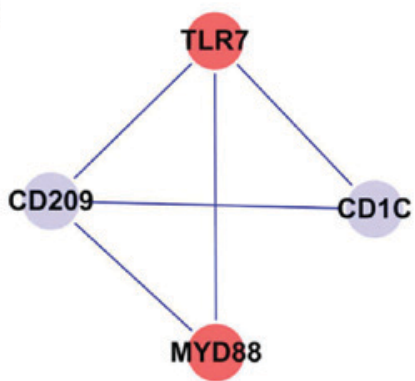

Figure 5. Sub-networks constructed based on the differentially expressed genes identified in the communities of interest in the protein-protein interaction network using CFinder software. The network is undirected, where a circular node represents a protein and a gray line represents an interaction between nodes. (A) A sub-PPI network was identified in the community 1 of the M1 group and is constructed of 42 protein nodes, 10 of which are colored red to indicate highest degree. (B) The PPI network consists of 11 proteins from community 1 of the M2 group, including seven of the previously identified highest degree proteins and 24 PPIs. (C) The PPI network derives from community 3 of the M2 group. PPI, protein-protein interaction.

in the program and link macrophage polarization to crucial biological programs, including immune response and malignancy progression.

In a previous study of mouse bone marrow-derived macrophages, macrophages were mock transfected and their transcriptional products analyzed (37). IFN responses were the primary reactions identified at 5 and $24 \mathrm{~h}$ after transfection, and the expression levels of IFN-mediated proteins (IFIH1, IFIT2 and IFIT3), the GBP protein family (GBP1 and GBP5), the transcription factor STAT1 and cytokine CXCL10 were detected. Increased expression of $I R F I$ was additionally detected in the transcriptional products of macrophages stimulated by IFN- $\gamma$ (37).

LPS, as well as IFN- $\gamma$ and TNF Th1-type cytokines, mediate the classical macrophage activation pathway (33). It has been demonstrated that LPS mediates the expression of IFIH1, GBP5, IRF1, IRF7, IFIT2, IFIT3 and CXCL10 and that their expression is due to pro-inflammatory responses of M1 macrophages. The expression of a number of genes stimulated by LPS is mediated by IFN- $\gamma$ and not IL- 4 or IL-10. These genes include GBP5, IFIH1, IFIT2, IFIT3 and STAT1 (38). During the replication of Orientia tsutsugamushi in macrophages, the expression of genes involved in M1 macrophage polarization is upregulated. When macrophages are infected by Orientia tsutsugamushi, the expression of IFN-stimulated genes, including CXCL10, IRF7 and ISG15, is increased (39). Exposure to LPS increases blood TNF levels via the canonical TLR4-associated $\mathrm{NF}-\kappa \mathrm{B}$ signaling pathway, resulting in inflammation. TLR4 recognizes LPS in the canonical NF- $\kappa \mathrm{B}$ signaling pathway and initiates a signaling cascade. This leads to the activation of $\mathrm{NF}-\kappa \mathrm{B}$ and the expression of pro-inflammatory cytokines. Therefore, the complex transcriptional programme induced in macrophages following LPS stimulation is a product of the coordinated action of the transcription factors (40), and inhibition of the TLR4-NF- $\kappa \mathrm{B}$ signaling pathway may shift M1 macrophage polarization toward the M2 phenotype.

The TLR signaling pathway upregulates the expression of pro-inflammatory gene products by activating STAT1 and $\mathrm{NF}-\kappa \mathrm{B}(41)$, thereby regulating M1 macrophage polarization. STAT1 has been demonstrated to be an important regulator of the biological responses of different TLRs. The expression of inflammatory cytokines mediated by TLR2, TLR4,
TLR7, TLR8 or TLR9 is suppressed in STAT1-deficient macrophages (42). In addition, activation of IRF3 is selectively coupled to TLR3 and TLR4 (43). Previous studies have demonstrated that IFN is involved in the regulation of TLR-triggered gene expression, including the TLR4 upregulation of IRF7 expression through TLR-, activator- and interferon-mediated signaling pathways and the upregulation of IRFI expression through TLR9 $(44,45)$. The present results further suggested that TLR7 serves as a mediator of M2 macrophage polarization. In contrast to the well-known macrophage polarization-associated TLR transmembrane family members, including TLR4, TLR7 is localized in the endosomal compartment, along with TLR3/9, and is associated with the viral-induced immune response (46). A recent study demonstrated that Hepatitis C virus-induced TLR7 stimulation results in monocyte differentiation and M2 macrophage polarization (36), which is consistent with the present study and further suggests that TLR7 stimulated macrophage polarization associated with chronic liver disease pathogenesis.

A previous study demonstrated that CDKN1A, or p21, serves an important role in the production of IL-1 $\beta$ and the progression of inflammatory diseases (47). CDKN1A-deficient mice are more prone to endotoxic shock, and CDKN1A-deficient macrophages produce more IL-1 $\beta$. CDKN1A was additionally demonstrated to suppress the stimulatory effect of macrophages on inflammatory stimulation factors, and CDKN1A-deficient macrophages produce more inflammatory response factors, which promote M1 macrophage polarization (47). In contrast, the presence of CDKN1A regulates M2 macrophage polarization. It was identified that CDKN1A inhibits the activation of macrophages by TLR (47). The carboxyl-terminal domain of CDKN1A inhibits macrophage function by enhancing protein kinase B phosphorylation, which suppresses p38 activation, thereby inhibiting the production of inflammatory cytokines in macrophages activated by TLR (48). Consequently, CDKN1A limits macrophage activation in inflammatory reactive diseases, including rheumatoid arthritis. Previous studies demonstrated that CDKN1A-deficient macrophages exhibit enhanced activation by TLR agonists compared with control macrophages, regardless of the study background $(47,49)$, which confirms the inhibitory effect of CDKN1A on TLR.

CCNA2 is essential for the initiation of DNA replication, transcription and regulation of the cell cycle and has been 
reported to be a key regulator of cell differentiation (50). It was reported that the expression of microRNA (miR)-125b is downregulated during macrophage TLR4 signaling (51). TLR4 activates macrophages to produce pro-inflammatory cytokines; whereas, mmu-miR-125b reduces the production of nitric oxide in activated macrophages, promotes the growth of tumor cells, and, at least partially, inhibits the expression of CCNA2 (52).

MYD88 is an adapter protein that transduces intracellular signals induced by TLRs and IL-1 receptors (IL-1Rs) and serves a critical role in TLR/IL-1R-mediated immune responses (53). It has been demonstrated that MYD88 is essential for most TLR signaling pathways (54) and serves an important role in the activation of the signaling pathways induced by all TLRs/IL-1Rs (except TLR3) (55). Therefore, MYD88 is a suitable target for abnormal regulation of the TLR/IL-1R signaling pathways under pathological conditions $(56,57)$. The absence of MYD88 in macrophages leads to a reduction in pro-inflammatory cytokine production mediated by TLR2, TLR5, TLR7 and TLR9 (58-61).

In conclusion, the present study used bioinformatics analysis to demonstrate that the TLR signaling pathway serves an important role in the regulation of macrophage polarization and that the high-degree proteins in the PPI network involved in molecular regulation of macrophage polarization are closely associated with proteins of the TLR signaling pathway, suggesting that the TLR signaling pathway will be an important direction for future studies of macrophage function in a systematic view. The aforementioned proteins that were identified may serve as a primary focus and may provide a useful reference for the intervention and regulation of macrophage polarization.

\section{Acknowledgements}

Not applicable.

\section{Funding}

The present study was supported by National Natural Science Foundation of China (grant nos. 81570579, 81602337 and 81702564).

\section{Availability of data and materials}

The datasets used and analyzed during the current study are available from the corresponding author on reasonable request.

\section{Authors' contributions}

BM, YY, ZL, DZ and WZ analyzed the Gene Expression Omnibus database, conducted the cluster analysis of differentially expressed genes and constructed the protein-protein interaction networks. BM and YY were primary contributors in writing the manuscript. YJ and DX conceived the study. All authors read and approved the final version of the manuscript.

\section{Ethics approval and consent to participate}

Not applicable.

\section{Patient consent for publication}

Not applicable.

\section{Competing interests}

The authors declare that they have no competing interests.

\section{References}

1. Murray PJ: Macrophage polarization. Annu Rev Physiol 79: 541-566, 2017.

2. Wynn TA, Chawla A and Pollard JW: Macrophage biology in development, homeostasis and disease. Nature 496: 445-455, 2013.

3. Murray PJ and Wynn TA: Protective and pathogenic functions of macrophage subsets. Nat Rev Immunol 11: 723-737, 2011.

4. O'Shea JJ and Murray PJ: Cytokine signaling modules in inflammatory responses. Immunity 28: 477-487, 2008.

5. Vergadi E, Ieronymaki E, Lyroni K, Vaporidi K and Tsatsanis C: Akt signaling pathway in macrophage activation and M1/M2 polarization. J Immunol 198: 1006-1014, 2017.

6. Mantovani A, Biswas SK, Galdiero MR, Sica A and Locati M: Macrophage plasticity and polarization in tissue repair and remodelling. J Pathol 229: 176-185, 2013.

7. Grailer JJ, Haggadone MD, Sarma JV, Zetoune FS and Ward PA: Induction of M2 regulatory macrophages through the $\beta 2$-adrenergic receptor with protection during endotoxemia and acute lung injury. J Innate Immun 6: 607-618, 2014.

8. Labonte AC, Tosello-Trampont AC and Hahn YS: The role of macrophage polarization in infectious and inflammatory diseases. Mol Cells 37: 275-285, 2014.

9. Incio J, Tam J, Rahbari NN, Suboj P, McManus DT, Chin SM, Vardam TD, Batista A, Babykutty S, Jung K, et al: PIGF/VEGFR-1 signaling promotes macrophage polarization and accelerated tumor progression in obesity. Clin Cancer Res 22: 2993-3004, 2016.

10. Castoldi A, Naffah de Souza C, Câmara NO and Moraes-Vieira PM: The macrophage switch in obesity development. Front Immunol 6: 637, 2016.

11. Kralova Lesna I, Petras M, Cejkova S, Kralova A, Fronek J, Janousek L, Thieme F, Tyll T and Poledne R: Cardiovascular disease predictors and adipose tissue macrophage polarization: Is there a link? Eur J Prev Cardiol 25: 328-334, 2018.

12. Fuentes-Duculan J, Suarez-Fariñas M, Zaba LC, Nograles KE, Pierson KC, Mitsui H, Pensabene CA, Kzhyshkowska J, Krueger JG and Lowes MA: A subpopulation of CD163-positive macrophages is classically activated in psoriasis. J Invest Dermatol 130: 2412-2422, 2010.

13. Beyer M, Mallmann MR, Xue J, Staratschek-Jox A, Vorholt D, Krebs W, Sommer D, Sander J, Mertens C, Nino-Castro A, et al: High-resolution transcriptome of human macrophages. PLoS One 7: e45466, 2012.

14. Bolstad BM, Irizarry RA, Astrand M and Speed TP: A comparison of normalization methods for high density oligonucleotide array data based on variance and bias. Bioinformatics 19: 185-193, 2003.

15. Marabita F, Almgren M, Lindholm ME, Ruhrmann S, Fagerström-Billai F, Jagodic M, Sundberg CJ, Ekström TJ, Teschendorff AE, Tegnér J and Gomez-Cabrero D: An evaluation of analysis pipelines for DNA methylation profiling using the Illumina HumanMethylation450 BeadChip platform. Epigenetics 8: 333-346, 2013.

16. Smyth GK, Michaud J and Scott HS: Use of within-array replicate spots for assessing differential expression in microarray experiments. Bioinformatics 21: 2067-2075, 2005.

17. Vallejos CA, Marioni JC and Richardson S: BASiCS: Bayesian analysis of single-cell sequencing data. PLoS Comput Biol 11: e1004333, 2015.

18. Warnes GR: gplots: Various R Programming Tools for Plotting Data. Version 3.0.1. https://cran.r-project.org/web/packages/ gplots/index.html. Accessed March 30, 2016.

19. Shannon P, Markiel A, Ozier O, Baliga NS, Wang JT, Ramage D, Amin N, Schwikowski B and Ideker T: Cytoscape: A software environment for integrated models of biomolecular interaction networks. Genome Res 13: 2498-2504, 2003. 
20. Palla G, Derényi I, Farkas I and Vicsek T: Uncovering the overlapping community structure of complex networks in nature and society. Nature 435: 814-818, 2005.

21. Sica A and Mantovani A: Macrophage plasticity and polarization: In vivo veritas. J Clin Invest 122: 787-795, 2012.

22. Komohara Y, Fujiwara Y, Ohnishi K, Shiraishi D and Takeya M: Contribution of macrophage polarization to metabolic diseases. J Atheroscler Thromb 23: 10-17, 2016.

23. Tariq M,Zhang J,Liang G, Ding L, He Q and Yang B: Macrophage polarization: Anti-cancer strategies to target tumor-associated macrophage in breast cancer. J Cell Biochem 118: 2484-2501, 2017.

24. Shapouri-Moghaddam A, Mohammadian S, Vazini H, Taghadosi M, Esmaeili SA, Mardani F, Seifi B, Mohammadi A, Afshari JT and Sahebkar A: Macrophage plasticity, polarization, and function in health and disease. J Cell Physiol 233: 6425-6440, 2018.

25. Mosser DM and Edwards JP: Exploring the full spectrum of macrophage activation. Nat Rev Immunol 8: 958-969, 2008.

26. Geissmann F, Manz MG, Jung S, Sieweke MH, Merad M and Ley K: Development of monocytes, macrophages, and dendritic cells. Science 327: 656-661, 2010.

27. Noel W, Raes G, Hassanzadeh Ghassabeh G, De Baetselier P and Beschin A: Alternatively activated macrophages during parasite infections. Trends Parasitol 20: 126-133, 2004.

28. Davies LC, Jenkins SJ, Allen JE and Taylor PR: Tissue-resident macrophages. Nat Immunol 14: 986-995, 2013.

29. Cui G, Kim B, Alguwaizani S and Han K: Assessing protein-protein interactions based on the semantic similarity of interacting proteins. Int J Data Min Bioinform 13: 75-83, 2015.

30. Yu L, Gao L and Sun PG: A hybrid clustering algorithm for identifying modules in Protein-Protein Interaction networks. Int J Data Min Bioinform 4: 600-615, 2010.

31. Ullah MO, Sweet MJ, Mansell A, Kellie S and Kobe B TRIF-dependent TLR signaling, its functions in host defense and inflammation, and its potential as a therapeutic target. J Leukoc Biol 100: 27-45, 2016.

32. Tong AJ, Liu X, Thomas BJ, Lissner MM, Baker MR, Senagolage MD, Allred AL, Barish GD and Smale ST: A stringent systems approach uncovers gene-specific mechanisms regulating inflammation. Cell 165: 165-179, 2016.

33. Zhou D, Huang C, Lin Z, Zhan S, Kong L, Fang C and Li J: Macrophage polarization and function with emphasis on the evolving roles of coordinated regulation of cellular signaling pathways. Cell Signal 26: 192-197, 2014.

34. Bonizzi G and Karin M: The two NF-kappaB activation pathways and their role in innate and adaptive immunity. Trends Immunol 25: 280-288, 2004.

35. Porta C, Rimoldi M, Raes G, Brys L, Ghezzi P, Di Liberto D, Dieli F, Ghisletti S, Natoli G, De Baetselier P, et al: Tolerance and M2 (alternative) macrophage polarization are related processes orchestrated by p50 nuclear factor kappaB. Proc Natl Acad Sci USA 106: 14978-14983, 2009.

36. Saha B, Kodys K, Adejumo A and Szabo G: Circulating and exosome-packaged hepatitis C single-stranded RNA induce monocyte differentiation via TLR7/8 to polarized macrophages and fibrocytes. J Immunol 198: 1974-1984, 2017.

37. Lacaze P, Raza S, Sing G, Page D, Forster T, Storm P, Craigon M, Awad T, Ghazal P and Freeman TC: Combined genome-wide expression profiling and targeted RNA interference in primary mouse macrophages reveals perturbation of transcriptional networks associated with interferon signalling. BMC Genomics 10: 372, 2009.

38. Zhang S, Kim CC, Batra S, McKerrow JH and Loke P: Delineation of diverse macrophage activation programs in response to intracellular parasites and cytokines. PLoS Negl Trop Dis 4: e648, 2010.

39. Tantibhedhyangkul W, Prachason T, Waywa D, El Filali A, Ghigo E, Thongnoppakhun W, Raoult D, Suputtamongkol Y, Capo C, Limwongse C and Mege JL: Orientia tsutsugamushi stimulates an original gene expression program in monocytes: Relationship with gene expression in patients with scrub typhus. PLoS Negl Trop Dis 5: e1028, 2011.

40. Medzhitov R and Horng T: Transcriptional control of the inflammatory response. Nat Rev Immunol 9: 692-703, 2009.

41. Smale ST: Selective transcription in response to an inflammatory stimulus. Cell 140: 833-844, 2010.

42. Kim HS, Kim DC, Kim HM, Kwon HJ, Kwon SJ, Kang SJ, Kim SC and Choi GE: STAT1 deficiency redirects IFN signalling toward suppression of TLR response through a feedback activation of STAT3. Sci Rep 5: 13414, 2015.
43. Smale ST and Natoli G: Transcriptional control of inflammatory responses. Cold Spring Harb Perspect Biol 6: a016261, 2014.

44. Battistini A: Interferon regulatory factors in hematopoietic cell differentiation and immune regulation. J Interferon Cytokine Res 29: 765-780, 2009.

45. Aly S, Mages J, Reiling N, Kalinke U, Decker T, Lang R and Ehlers S: Mycobacteria-induced granuloma necrosis depends on IRF-1. J Cell Mol Med 13: 2069-2082, 2009.

46. Petes C, Odoardi N and Gee K: The toll for trafficking: Toll-like receptor 7 delivery to the endosome. Front Immunol 8: 1075, 2017.

47. Scatizzi JC, Mavers M, Hutcheson J, Young B, Shi B, Pope RM, Ruderman EM, Samways DS, Corbett JA, Egan TM and Perlman H: The CDK domain of p21 is a suppressor of IL-1beta-mediated inflammation in activated macrophages. Eur J Immunol 39: 820-825, 2009.

48. Mavers M, Cuda CM, Misharin AV, Gierut AK, Agrawal H, Weber E, Novack DV, Haines GK III, Balomenos D and Perlman H: Cyclin-dependent kinase inhibitor p21, via its C-terminal domain, is essential for resolution of murine inflammatory arthritis. Arthritis Rheum 64: 141-152, 2012.

49. Trakala M, Arias CF, Garcia MI, Moreno-Ortiz MC, Tsilingiri K, Fernández PJ, Mellado M, Díaz-Meco MT, Moscat J, Serrano M, et al: Regulation of macrophage activation and septic shock susceptibility via p21(WAF1/CIP1). Eur J Immunol 39: 810-819, 2009.

50. Anger M, Bryja V, Jirmanova L, Hampl A, Carrington M, Motlik J, Dvorak P and Kubelka M: The appearance of truncated cyclin A2 correlates with differentiation of mouse embryonic stem cells. Biochem Biophys Res Commun 302: 825-830, 2003

51. Tili E, Michaille JJ, Cimino A, Costinean S, Dumitru CD, Adair B, Fabbri M, Alder H, Liu CG, Calin GA and Croce CM: Modulation of miR-155 and miR-125b levels following lipopolysaccharide/TNF-alpha stimulation and their possible roles in regulating the response to endotoxin shock. J Immunol 179: 5082-5089, 2007 .

52. Xu Z, Zhao L, Yang X, Ma S, Ge Y, Liu Y, Liu S, Shi J and Zheng D: Mmu-miR-125b overexpression suppresses NO production in activated macrophages by targeting eEF2K and CCNA2. BMC Cancer 16: 252, 2016.

53. Deguine J and Barton GM: MyD88: A central player in innate immune signaling. F1000Prime Rep 6: 97, 2014.

54. O'Neill LA and Bowie AG: The family of five: TIR-domain-containing adaptors in Toll-like receptor signalling. Nat Rev Immunol 7: 353-364, 2007.

55. Alexopoulou L, Holt AC, Medzhitov R and Flavell RA: Recognition of double-stranded RNA and activation of NF-kappaB by Toll-like receptor 3. Nature 413: 732-738, 2001.

56. Loiarro M, Ruggiero V and Sette C: Targeting TLR/IL-1R signalling in human diseases. Mediators Inflamm 2010: 674363, 2010.

57. Loiarro M, Ruggiero V and Sette C: Targeting the Toll-like receptor/interleukin 1 receptor pathway in human diseases: Rational design of MyD88 inhibitors. Clin Lymphoma Myeloma Leuk 13: 222-226, 2013.

58. Hayashi F, Smith KD, Ozinsky A, Hawn TR, Yi EC, Goodlett DR, Eng JK, Akira S, Underhill DM and Aderem A: The innate immune response to bacterial flagellin is mediated by Toll-like receptor 5. Nature 410: 1099-1103, 2001.

59. Hemmi H, Kaisho T, Takeuchi O, Sato S, Sanjo H, Hoshino K, Horiuchi T, Tomizawa H, Takeda $\mathrm{K}$ and Akira S: Small anti-viral compounds activate immune cells via the TLR7 MyD88-dependent signaling pathway. Nat Immunol 3: 196-200, 2002.

60. Schnare M, Holt AC, Takeda K, Akira S and Medzhitov R: Recognition of CpG DNA is mediated by signaling pathways dependent on the adaptor protein MyD88. Curr Biol 10: $1139-1142,2000$

61. Takeuchi O, Kaufmann A, Grote K, Kawai T, Hoshino K, Morr M, Mühlradt PF and Akira S: Cutting edge: Preferentially the R-stereoisomer of the mycoplasmal lipopeptide macrophage-activating lipopeptide- 2 activates immune cells through a toll-like receptor 2- and MyD88-dependent signaling pathway. J Immunol 164: 554-557, 2000.

This work is licensed under a Creative Commons Attribution-NonCommercial-NoDerivatives 4.0 International (CC BY-NC-ND 4.0) License. 